\title{
Sample size for detecting differentially expressed genes in microarray experiments Caimiao Wei ${ }^{1}$, Jiangning $\mathrm{Li}^{2}$ and Roger E Bumgarner*1
}

Address: ${ }^{1}$ Department of Microbiology, University of Washington, Seattle, WA 98195, USA and 2Department of Pathology, University of Washington, Seattle, WA 98195, USA

Email: Caimiao Wei - caimiaow@u.washington.edu; Jiangning Li - ljn68@u.washington.edu; Roger E Bumgarner* - rogerb@u.washington.edu

* Corresponding author

Published: 08 November 2004

BMC Genomics 2004, 5:87 doi:10.1 |86/|47|-2/64-5-87
Received: 14 June 2004

Accepted: 08 November 2004

This article is available from: http://www.biomedcentral.com/I47I-2/64/5/87

(c) 2004 Wei et al; licensee BioMed Central Ltd.

This is an Open Access article distributed under the terms of the Creative Commons Attribution License (http://creativecommons.org/licenses/by/2.0), which permits unrestricted use, distribution, and reproduction in any medium, provided the original work is properly cited.

\begin{abstract}
Background: Microarray experiments are often performed with a small number of biological replicates, resulting in low statistical power for detecting differentially expressed genes and concomitant high false positive rates. While increasing sample size can increase statistical power and decrease error rates, with too many samples, valuable resources are not used efficiently. The issue of how many replicates are required in a typical experimental system needs to be addressed. Of particular interest is the difference in required sample sizes for similar experiments in inbred vs. outbred populations (e.g. mouse and rat vs. human).

Results: We hypothesize that if all other factors (assay protocol, microarray platform, data preprocessing) were equal, fewer individuals would be needed for the same statistical power using inbred animals as opposed to unrelated human subjects, as genetic effects on gene expression will be removed in the inbred populations. We apply the same normalization algorithm and estimate the variance of gene expression for a variety of cDNA data sets (humans, inbred mice and rats) comparing two conditions. Using one sample, paired sample or two independent sample t-tests, we calculate the sample sizes required to detect a 1.5-, 2-, and 4-fold changes in expression level as a function of false positive rate, power and percentage of genes that have a standard deviation below a given percentile.

Conclusions: Factors that affect power and sample size calculations include variability of the population, the desired detectable differences, the power to detect the differences, and an acceptable error rate. In addition, experimental design, technical variability and data pre-processing play a role in the power of the statistical tests in microarrays. We show that the number of samples required for detecting a 2 -fold change with $90 \%$ probability and a p-value of 0.01 in humans is much larger than the number of samples commonly used in present day studies, and that far fewer individuals are needed for the same statistical power when using inbred animals rather than unrelated human subjects.
\end{abstract}

\section{Background}

Microarray technology has become an important tool for studying gene expression levels on the whole genome scale [1]. One important objective of many microarray studies is to identify differentially expressed genes between different conditions. Despite the effectiveness of 
the technology, microarray experiments are usually done with very few replicates due to budgetary constrains, which often results in high false positive (Type I error) and false negative rates (Type II error). For many microarray experiments, once a list of genes has been identified, intensive follow-up investigations of these genes using traditional molecular tools are often pursued. Hence, valuable resources can be wasted in pursuing genes from experiments with a high false positive rate. Increasing the sample size increases the statistical power to detect expression differences in microarray analysis while also decreasing the error rate. However, it is important to balance sample size with other experimental goals so as not to waste resources. An important issue that concerns many biologists is, therefore "how many replicates are needed to obtain a given type of result?"

In general, the required sample size depends on the magnitude of the variability of the population, the magnitude of the expression change that is biologically meaningful (or desirable to detect), the power to detect the expression change, and the P-value/significance level/false positive rate. However, power and sample size have been viewed as complicated and difficult issues for microarray studies due to the large number of genes being investigated and little knowledge of the degree of natural expression variation within a population. To date, very few studies have assessed power and sample size requirements in microarray experiments. Pan et al. [2] proposed a normal mixture model to calculate the number of replicates required. In this study, the parameters were estimated using a subset of a real data set generated by cDNA arrays. This paper assumed that the replicates were independent of each other, whether they were drawn from the same individual or multiple individuals. Lee and Whitmore [3] discussed conceptual issues and presented computational methods (Analysis of Variance) for statistical power and sample size for different types of experimental designs, taking multiple testing into account. However, the data sets used to demonstrate these models contained a single pooled sample for each treatment/time point but not true biological replicates. Zien et al. [4] proposed a complex model that applies only to Affymetrix data to estimate biological variation and measurement error (normal additive and multiplication measurement error) for two sample comparisons. This study was based on 5 real Affymetrix data sets where the minimum required sample size was estimated based on a simulation study. Zien et al. [4] assumed a normal distributed additive measurement error and lognormal distributed measurement error. However, in real data, the functional form of the distribution of gene expression levels is generally unknown. The most common practice in microarray experiments is to assume normality of log transformed intensities or ratios. Pavlidis et al. [5] used a random sampling approach to evaluate the stability of the genes found to be differentially expressed between two groups from 16 published data sets; they found that the stability of some of the smaller data sets with fewer than 10 replicates was inconclusive. This approach is sound for the purpose of planning a study when pilot data is available with a large number of replicates. However, a pilot study is usually done with a small number of replicates where this is not feasible.

Pair-wise comparisons between conditions/groups/treatments are frequently used in microarray studies. Parametric and nonparametric statistical methods have been proposed to identify differentially expressed genes, among which t-tests are most commonly used. This paper is intended to provide some guidelines for sample size planning for pair-wise comparisons. Normalization is an essential and important pre-processing step in microarray data analysis. To our knowledge, no previous studies using multiple data sets have pre-processed the data sets in a comparable way. In addition, previous studies did not look at the effect of inbred vs outbred populations on the variation of gene expression. In order to make the results more comparable, we make use of 7 cDNA microarray data sets and apply the same normalization method (spatial lowess).

We estimate the variance by one sample t-test, paired $\mathrm{t}$ tests or two sample t-tests on a gene-by-gene basis using several large expression data sets from both human, rats and mice. We then calculate the sample size required to detect a 1.5-, 2-, and 4-fold change in expression levels for the $90^{\text {th }}, 75^{\text {th }}, 50^{\text {th }}$ and $25^{\text {th }}$ percentile of genes ranked by variability at fixed settings for false positive and false negative rates. The sample size calculation provides the approximate but not exact number of replicates required for a given set of criteria.

\section{Results \\ Data sets}

We estimate the standard deviation and required sample size from 1 unpublished and 6 published cDNA data sets (Table 1). Data set A-C and E are from human samples. Data sets D and F-G are from mouse or rat samples. Data set $\mathrm{F}$ is from a study that is not yet published. The raw and/ or pre-processed data for the unpublished dataset with gene order randomized and without the original gene/ probe identifications can be downloaded from http:// expression.washington.edu/publications/caimiao/ power\&samplesize/samplesize.html. Removing the gene IDs does not affect the analysis in this paper in any way. The same website also provides the links for the published data sets. In addition, all the control genes were removed from the data analysis for the cDNA data sets. 
Table I: cDNA microarray data sets used in the study

\begin{tabular}{|c|c|c|c|c|c|c|}
\hline Data set & Reference & \# Rep & \# Genes & Tissue type & Description & Hybridization \\
\hline$A$ & Smith et al. 2003 & 20 & 15,592 & Human liver & $\begin{array}{l}\text { Paired HCC tumor vs } \\
\text { adjacent non-tumor }\end{array}$ & $\begin{array}{l}\text { Direct hyb between } \\
\text { tumor and non tumor }\end{array}$ \\
\hline B & Lapointe et al. 2004 & 41 & 38627 & Human prostate & $\begin{array}{l}\text { Paired prostate tumor } \\
\text { vs adjacent non-tumor }\end{array}$ & $\begin{array}{l}\text { Indirect hyb using } \\
\text { common reference }\end{array}$ \\
\hline C & Chen et al 2002 & 48 & 22618 & Human liver & $\begin{array}{c}\text { Paired } \mathrm{HCC}+\mathrm{HBV} \text { vs } \\
\mathrm{HBV}\end{array}$ & $\begin{array}{l}\text { Indirect hyb using } \\
\text { common reference }\end{array}$ \\
\hline $\mathrm{D}$ & Pritchard et al. $200 \mathrm{I}$ & 6 & 5281 & Mouse liver and kidney & Paired liver vs kidney & $\begin{array}{l}\text { Indirect hyb using } \\
\text { common reference }\end{array}$ \\
\hline E & Zhao et al. 2004 & 36 ductal +21 lobular & 44549 & Human breast & $\begin{array}{l}\text { lobular and ductal tumor } \\
\text { tissue }\end{array}$ & $\begin{array}{l}\text { Indirect hyb using } \\
\text { common reference }\end{array}$ \\
\hline $\mathrm{F}$ & NA & 6 & 13,056 & Mouse liver & $\begin{array}{l}\text { One third vs two thirds } \\
\text { hepatectomy }\end{array}$ & $\begin{array}{l}\text { Indirect hyb using } \\
\text { individual baseline }\end{array}$ \\
\hline G & Callow et al. 2000 & 8 & 5548 & Mouse liver & $\begin{array}{c}\text { ApoAl knock-out vs } \\
\text { normal }\end{array}$ & $\begin{array}{l}\text { Indirect hyb using } \\
\text { common reference } \\
\text { (pool) }\end{array}$ \\
\hline
\end{tabular}

Data set A comprises data generated from 40 liver RNA samples isolated from paired liver hepatocellular carcinoma (HCC) tumor and adjacent cirrhotic non-tumor tissue from $20 \mathrm{HCV}$ infected Caucasian patients [6]. The objective of this study was to identify potential hepatocellular carcinoma markers. The microarray analysis was performed using in-house spotted human cDNA arrays containing 15,592 genes split between and spotted in duplicate on arrays HHD1 and HHD2. The duplicate sets of cDNAs within each slide were spotted side by side (panels A, B). RNA from tumor and adjacent non-tumor tissue from individual patients was co-hybridized to 2 slides (one was a dye flip of the other).

Data set B was generated from 41 matched pairs of prostate tumor and non-tumor tissue hybridized to arrays spotted with 38627 cDNAs. All samples were labeled with Cy5 and co-hybridized with a common reference labeled in Cy3 [7]. The purpose of this study was to identify the difference in expression levels between normal and prostate tumor tissues.

Data set $\mathrm{C}$ was generated from RNA isolated from paired HCC tumor and adjacent non tumor liver from $41 \mathrm{HBV}$ infected patients $[8,9]$ hybridized to cDNA arrays. Each RNA sample was labeled with either Cy5 and co-hybridized with Cy3-labeled reference RNA. The purpose of this study was to identify gene expression differences between HCC tumor and non-tumor liver tissue.

Data set D was generated from RNA isolated from paired liver and kidney tissue from 6 male C57BL6 mice [9] hybridized to cDNA arrays. Each RNA sample was labeled with Cy5 and co-hybridized with Cy3-labeled amplified
RNA from Universal Human Reference to total RNA (Stratagene). The purpose of this study was to identify gene expression differences between tissue types.

Data set E was generated from RNA isolated from 36 breast ductal tumor and 16 lobular tumor tissues [10] hybridized to cDNA arrays. Each RNA sample was split in two and labeled with either Cy5 or Cy3, and co-hybridized with a common reference RNA as color flips with two replicates (4 arrays/tissue/mouse). The purpose of this study was to identify gene expression differences between ductal carcinoma and lobular carcinoma.

Data set $\mathrm{F}$ consists of data generated from 24 liver tissue samples from 12 inbred mice (unpublished data). One third or two thirds of the liver was removed from each mouse and used as the baseline samples. At 12 hours post operation, the mice were sacrificed and the remaining liver tissue was used as the experimental sample. The aim of this study was to screen for genes potentially related to liver regeneration after hepatectomy. RNA samples from the 12 hour post-operation livers were co-hybridized with their own baseline liver samples. A total of four DNA arrays were used for each sample comparison. Two sets of arrays (MOD1 and MOD2), each containing 6528 different cDNAs spotted in duplicate (A and B) on each array were used. In addition, each comparison was done with a dye flip pair of slides. This data set made use of arrays generated at the University of Washington Center for Expression Arrays.

The goal of data set G was to identify genes with altered expression in the liver tissues of two mouse models with very low HDL cholesterol levels (treatment groups) as 
compared to inbred control mice. The mouse model considered in this study is the Apolipoprotein AI (ApoAI) knock-out, where ApoAI is a gene known to play a pivotal roles in HDL metabolism $[11,12]$. Each cDNA array contained 5548 non-control genes or ESTs. A pool of normal RNA samples labeled with Cy3 served as the reference for all the arrays.

In summary, three of the data sets (D, F-G) are from inbred mouse and rat strains respectively, and the other four data sets (A-C, and E) are from large scale studies of gene expression in humans. If all other factors (assay protocol microarray platform, data pre-processing) were equal, one might anticipate that fewer individuals would be needed for the same statistical power using inbred animals as opposed to unrelated human subjects.

\section{Background adjustment and normalization}

Background adjustment and normalization is necessary to remove systematic biases of non-biological origin in microarray studies. A number of methods of background correction and normalization have been proposed $[13,14]$. We used the locally written program "spot-on Image" to analyze the cDNA array data for data sets A and F. Spot-on uses a local background for each spot. The background subtracted intensity of all cDNA data sets were normalized by the spatial lowess method using the $\mathrm{R}$ add on package MAANOVA written by the Jackson Lab, which is available at http://www.jax.org/staff/churchill/ labsite/software/index.html.

\section{Estimates of standard deviation and sample size calculation}

The distribution of the standard deviations estimated from these 7 data sets are presented in Figure 1. All data are $\log _{2}$ transformed prior to data analysis. Figure $1 \mathrm{~A}$ shows the standard deviation of the log ratio of the 4 paired cDNA data sets (A-D). The standard deviations of data sets E-G in Figure 1B are the common standard deviation of the $\log _{2}$ ratio (sample/reference) of two independent groups.

The required sample size of an experiment depends on the variance component $(\sigma)$, the desired detectable fold change $(\delta)$, the power to detect this change (1- $\beta$, the likelihood of detecting the change or the true positive rate), and a chosen type I error rate $(\alpha)$. For microarrays, a combination of fold-change and test p-value is commonly used for selecting differentially expressed genes between two groups or conditions. In this study, sample sizes were calculated in $\mathrm{R}$ using the function of power.t.test. The required input parameters are the log scale fold change of interest $\delta$ ( $\delta=1$ in log transformed data translates into a 2 fold change in expression level, $\delta=2$ in log transformed data translates into a 4 fold change in expression level, etc), significance level, power, and the standard deviation (common standard deviation for two sample t-test, the standard deviation of the difference within subject for paired t-test, or the standard deviation of one sample ttest), the type of t-tests (one sample, two sample, or paired t-test), and the type of test (two sided or one sided).

For example, in the case of data set A (one sample t-test), if we wish to find out the approximate sample size to detect a 2 fold change $(\delta=1)$ in expression level between tumor and non-tumor tissue in the $75 \%$ least variable genes $(\sigma<=0.5884$ ) with a two sided 0.001 significance level test with $90 \%$ power, we could use the following $\mathrm{R}$ function

power.t.test $(\mathrm{n}=\mathrm{NULL}$, delta $=1, \mathrm{sd}=0.5584$, sig.level $=$ 0.001 , power $=0.9$, type $=$ "one.sample", alternative $=$ "two.sided")

Where $\mathrm{sd}=0.5584$ is the $75^{\text {th }}$ percentile of the standard deviation of $\log$ ratio.

In the case of data set $\mathrm{G}$ (two sample t-test), if we wish to find the approximate sample size to detect a 2 fold change $(\delta=1)$ in expression level between knock-out and control mice in the $75 \%$ least variable genes $(\sigma<=0.3102)$ with a two sided 0.001 significance level test with $90 \%$ power, we could use the following $\mathrm{R}$ function

power.t.test $(\mathrm{n}=\mathrm{NULL}$, delta $=1, \mathrm{sd}=0.3102$, sig.level $=$ 0.001 , power $=0.9$, type $=$ "two.sample", alternative $=$ "two.sided")

Where $\mathrm{sd}=0.3102$ is the $75^{\text {th }}$ percentile of the common standard deviation of log (sample/reference).

In $\mathrm{R}$, for a one sample t-test or a paired t-test to have power $1-\beta$ to reject for a two sided testing and strict interpretation of tail probability with significance level $\alpha$ for detecting a difference of $\delta$, the minimum number of samples or pairs is obtained by solving the following equation iteratively

Power $=\operatorname{Pr}\left(\mathrm{t}_{\mathrm{v}, \mathrm{ncp}}<\mathrm{t}_{\mathrm{v}, \alpha / 2}\right)+\operatorname{Pr}\left(\mathrm{t}_{\mathrm{v}, \mathrm{ncp}}>\mathrm{t}_{\mathrm{v}, 1-\alpha / 2}\right)$

Where ncp is the noncentrality parameter of the non-central $\mathrm{t}$-distribution, and is estimated by

$$
\mathrm{ncp}=\frac{\delta}{\sigma \sqrt{\frac{1}{n}}}
$$

$\mathrm{t}_{\mathrm{v}, \alpha / 2}$ is the $\alpha / 2$ quantile of a central t-distribution with $\mathrm{v}$ degrees of freedom and $\mathrm{v}=\mathrm{n}-1 . \mathrm{t}_{\mathrm{v}, \mathrm{ncp}}$ follows a non-central 


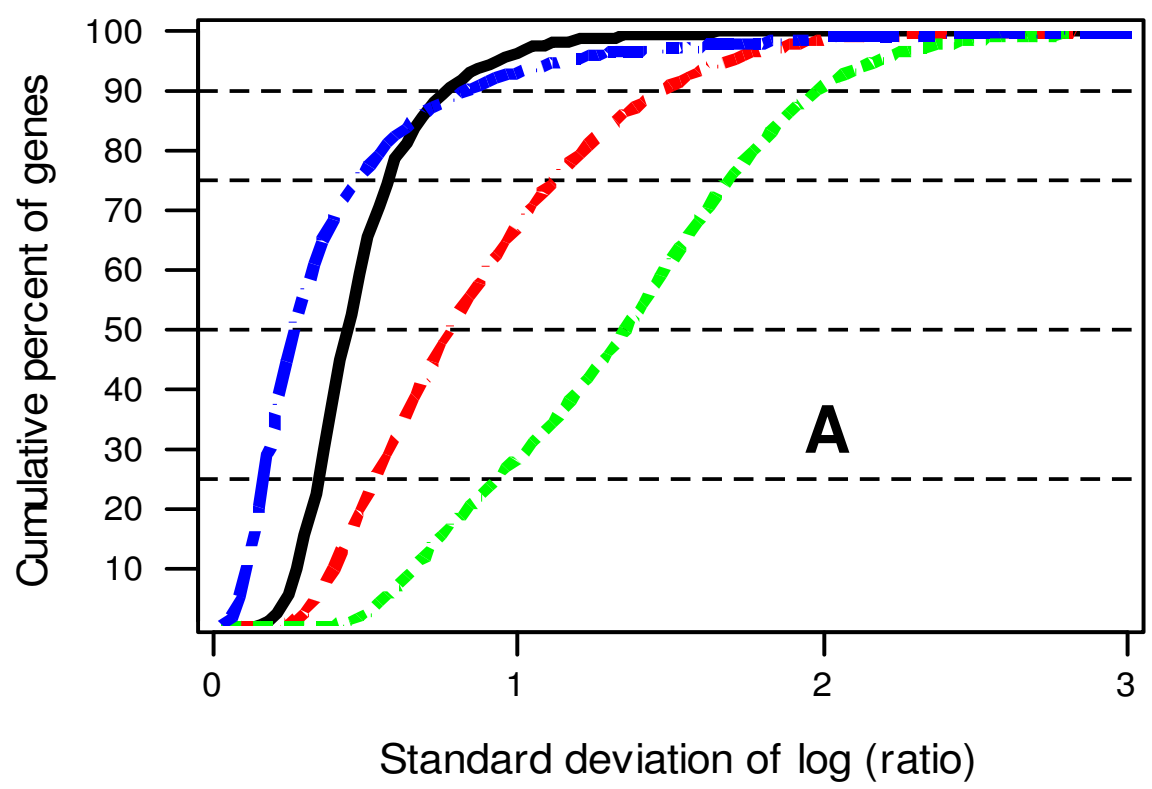

\begin{tabular}{|ll|}
\hline Black & Data set A \\
Red & Data set B \\
Green & Data set C \\
Blue & Data set D \\
\hline
\end{tabular}

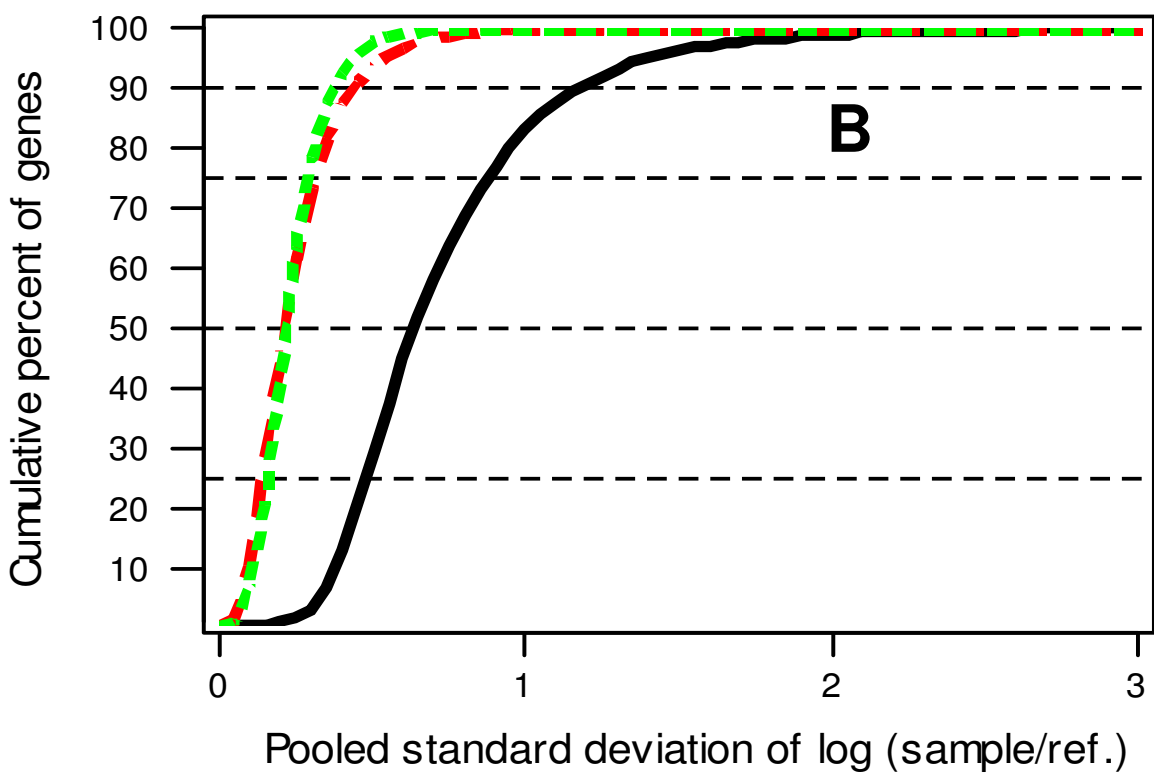

\begin{tabular}{|ll|}
\hline Black & Data set $\mathrm{E}$ \\
Red & Data set $\mathrm{F}$ \\
Green & Data set $\mathrm{G}$ \\
\hline
\end{tabular}

Figure I

Histogram of standard deviation The $X$ axis is the standard deviation, and the $Y$ axis is the percentage of genes that has standard deviation below the value of X. All data sets were normalized by spatial lowess; (A) Data set A-standard deviation of log ratio of two groups (direct hybridization); data set B-D standard deviation of the difference of log (sample/reference) of the two groups (indirect hybridization); (B) Data sets E-G common standard deviation of (sample/reference) of the two independent groups (indirection hybridization). 
t-distribution with $v$ degrees of freedom and a non-centrality parameter of ncp.

For a two sample t-test with equal sample sizes, if we wish to have a large enough sample to detect a difference $\delta$ (with a two-sided test and strict interpretation of tail probability with $\alpha$ significance level test with $1-\beta$ power), then the sample size (n) for each group is obtained by solving the following equation iteratively

Power $=\operatorname{Pr}\left(\mathrm{t}_{\mathrm{v}, \mathrm{ncp}}<\mathrm{t}_{\mathrm{v}, \alpha / 2}\right)+\operatorname{Pr}\left(\mathrm{t}_{\mathrm{v}, \mathrm{ncp}}>\mathrm{t}_{\mathrm{v}, 1-\alpha / 2}\right)$

Where ncp is the noncentrality parameter of non-central tdistribution, and is estimated by

$$
\mathrm{ncp}=\frac{\delta}{\sigma \sqrt{\frac{2}{n}}}
$$

$\mathrm{t}_{\mathrm{v}, \alpha / 2}$ is the $\alpha / 2$ quantile of a central t-distribution with $\mathrm{v}$ degrees of freedom and $v=2 n-2 . t_{v}$ ncp follows a non-central t-distribution with $\mathrm{v}$ degrees of freedom and a noncentrality parameter of ncp.

Microarray experiments usually involve a large number of genes, with variance components varying greatly across the genes. In general, the variance is higher for low expressors which make up of a large percentage of the genes (Figure 2). Approximately $50 \%$ of genes are called absent on the Affymetrix full genome GeneChips. It is reasonable to choose a value of variance, e.g. the median or the upper $75^{\text {th }}$ percentile of variance across all genes, and to use this as the value in the power calculations. For example, if we use the variance for the $50^{\text {th }}$ percentile, then the sample size calculations will assure us of having the desired power to detect a chosen n-fold change for all but the 50\% most variable genes. In Figure 1, we show horizontal lines at the $25^{\text {th }}, 50^{\text {th }}, 75^{\text {th }}$ and $90^{\text {th }}$ percentiles. The intersection of these lines with the "cumulative percentage of genes" provides the value of $\alpha$ for each data set. Additional file 1 shows the estimated sample size required to detect a 1.5-, 2-, and 4-fold change in expression level for the $90^{\text {th }}, 75^{\text {th }}, 50^{\text {th }}$, and $25^{\text {th }}$ percentile genes for a given setting of false positive rate and power. As is expected, the required sample size increases with increasing variance, increasing power, and decreasing foldchange and false positive rate.

A significance level (the probability of making a type I error, that is getting a false positive) of 0.05 is often employed in hypothesis testing. Thousands of genes are usually studied in microarray experiments. When more than 10,000 genes are tested independently, we would expect more than 500 genes to appear as false positives when the 0.05 significance level is applied. Hence, a smaller cut-off p-value should be used in order to reduce the number of false positives. Many multiple testing correction methods have been proposed. The simplest one is the Bonferroni correction (family wise error control) where the nominal significance level is divided by the number of tests. The Bonferroni correction is very stringent. False discovery rate (FDR) [15], the proportion of false positives among the genes that are identified as differentially expressed, is a post-data measure for controlling false positive. For the purpose of sample size planning, we suggest using the family wise type of error control. A higher false positive rate (lower false negative rate) can be employed for studies aiming to eliminating non-significant differentially expressed genes, and a smaller false positive rate can be used for those studies involving costly follow-up research.

For reference, Table 2 lists the number of genes/ESTs/ probes found to be differentially expressed between two conditions by different significance levels and fold changes. The number of significant genes in data sets $\mathrm{F}$ and $\mathrm{G}$ are small, possibly due to the relatively fewer number of genes included in the study and the homogeneity between conditions (data set F compare in-bred mice with two different volumes of hepatectomy; data set $\mathrm{G}$ compares ApoAI knock-out versus normal mice).

\section{Discussion}

Factors that affect sample size calculation include the magnitude of the variability of the population, the magnitude of the desired detectable expression change, the chosen power to detect the expression change, and the cut-off $\mathrm{P}$-value/significance level/false positive rate. For a given study, the variability of the population being studied is fixed, and once researchers have identified the desired detectable expression change, the required sample size depends on the chosen false positive and false negative rates. The variability of human subject data is typically larger than that seen with laboratory animals and cell lines due to genetic influences on gene expression. Hence, more replicates are needed for studies that involve human subjects (or any other outbred population) than for studies with samples from an inbred population. This is readily apparent in the cDNA data in Additional file 1 (data sets $\mathrm{A}-\mathrm{C}$, and $\mathrm{E}$ are human samples while $\mathrm{D}$ and $\mathrm{F}-\mathrm{G}$ are from mice). With the cDNA array data, one needs roughly 5 times as many human samples relative to mouse to detect the same magnitude of change with the same statistical power at the same significance level. This increase in the required number of samples for an outbred population has not been discussed before and has practical implications for those wishing to translate gene expression work from animal models to studies in human populations. 
Data set A

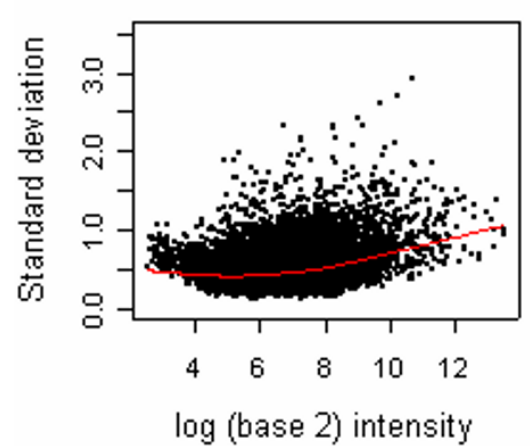

Data set D

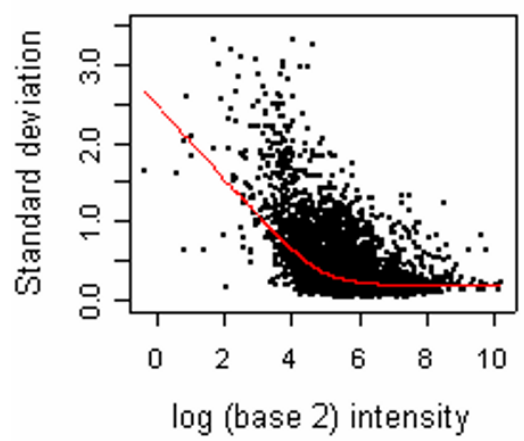

Data set G

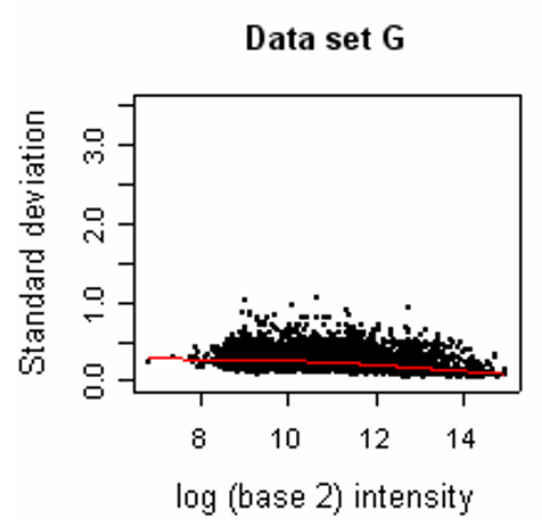

$\log$ (base 2) intensity
Data set B

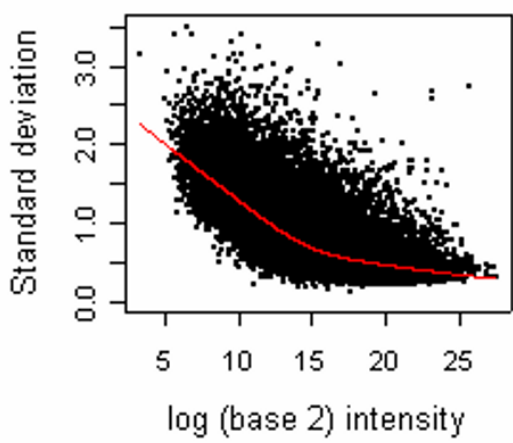

Data set $\mathrm{E}$

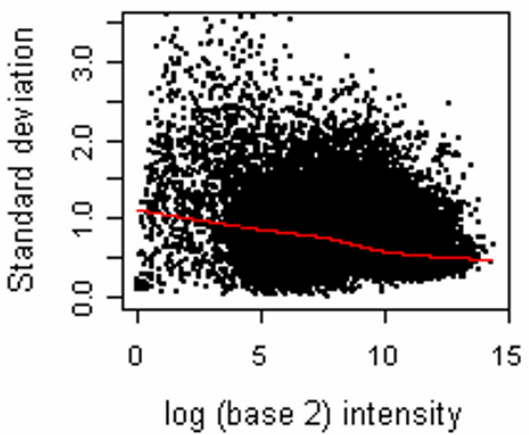

Data set C

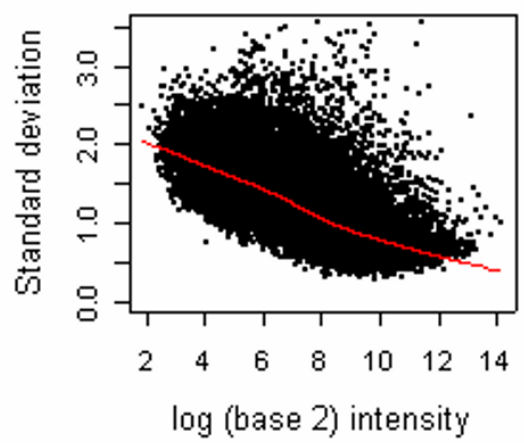

Data set $F$

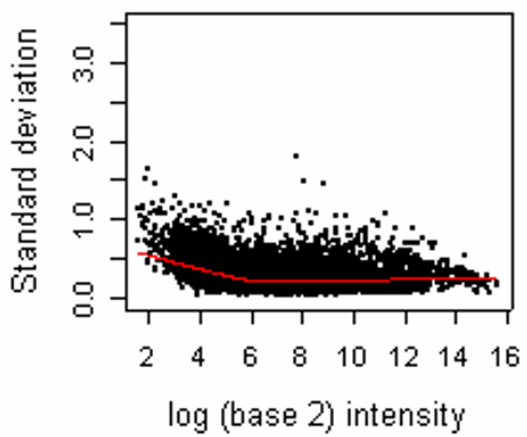

Figure 2

Standard deviation versus log intensity Standard deviations are based on one sample t-test (data set A), paired t-test (data sets $B-D)$, or two independent $t$-test (data sets $E-G$ ).

Multiple levels of replicates are common in two color microarray experiments. Multiple arrays probed with RNA samples isolated from multiple individuals of a population/treatment/group are referred to as biological replicates. Multiple arrays hybridized using the same RNA or multiple replicates of the same gene within an array are referred to as technical replicates. Although technical replicates can improve the precision and the reliability of the measurement and provide information for quality control, biological replicates are most effective in reducing the variance of the estimate of mean difference. Biological replicates therefore increase the power to detect 
Table 2: Significant genes/ESTs/probes called by methods used in the studies using different criteria (combination of significance level and fold changes)

\begin{tabular}{|c|c|c|c|c|}
\hline Data set & Reference & $\begin{array}{l}\mathrm{P}<=0.00 \mathrm{I} \text { and the } \\
\text { estimated fold change }>=2\end{array}$ & $P<=0.001$ only & $P<=0.01$ only \\
\hline$A$ & Smith et al. 2003 & 183 & 1783 & 3590 \\
\hline B & Lapointe et al. 2004 & 609 & 6549 & 10153 \\
\hline C & Chen et al 2002 & 1253 & 4187 & 6197 \\
\hline $\mathrm{D}$ & Pritchard et al. 200I & 479 & 1557 & 1845 \\
\hline$E$ & Zhao et al. 2004 & 270 & 1050 & 3821 \\
\hline $\mathrm{F}$ & NA & 16 & 145 & 723 \\
\hline$G$ & Callow et al. 2000 & 6 & II & 77 \\
\hline
\end{tabular}

biologically significant gene expression differences. More importantly, when trying to identify differences between a treatment and a control group, accurate estimates of the biological variability within the groups is essential to determine if the between group differences are meaningful (by a t-test, Analysis of Variance (ANOVA) or other method).

Careful experimental design is necessary to maximize the statistical power of the test [16-18] while balancing resource allocation. For example, dye swapping (in which pairs of RNA samples are hybridized twice with reverse dye labeling) is common in two color array experiments and is a great help in removing dye bias. However, if the experiments involve a common reference sample, which is not of biological interest and the goal is to identify gene expression differences between two groups (both of which are co-hybridized again the common reference), using twice as many independent biological replicates is preferable to dye swapped technical replicates.

\section{Caveats}

This paper is intended to give some guidance to those planning microarray experiments. The sample size calculations we performed provide an approximate number of replicates for a given set of criteria. Our studies were limited to a small number of published microarray studies for which the following criteria were true:

1) A reasonably large number of biological replicates were analyzed.

2) Raw data was readily available so that we could reprocess all data with the same algorithms.

3) Other potentially large sources of variability such as flow sorting, laser micro-dissection and/or multiple rounds of amplification were not present.

We have only analyzed date from a limited number of tissue types - liver, prostate, breast and blood in human, liver and kidney in mouse, and mammary gland in rat. It is entirely possible that different tissue types will have larger or smaller degrees of biological variation and hence will require more or fewer samples to reach a given conclusion. In addition, lab or experiment specific methods of obtaining and processing samples may induce greater degrees of expression variation than seen in our sample data. As more large data sets become available, it will be useful to extend these studies to better define the magnitude of gene expression variation in purebred animals and in outbred humans across a variety of tissues.

However, the data shown in Additional file 1 however should be sobering to those planning or reviewing an experimental protocol for microarray analysis. In these limited data sets with human samples hybridized to cDNA arrays with a common reference (B-C, E), we show that 32 samples for each group are required to detect a 2fold change in the $75 \%$ least variable genes with $90 \%$ power and a p-value of 0.001 . With a p-value of 0.01 and $90 \%$ power, at least 20 samples are required to detect a 2 fold change in the $75 \%$ least variable genes. This is a much larger number of samples than is frequently used in human microarray case-control studies designed to identify gene expression differences between two groups.

\section{Methods}

\section{Data set selection, pre-processing and normalization}

Background adjustment and normalization is needed in microarray data analysis in order to remove non-biological variation. Intensity based normalization methods such as locally weighted least square polynomial regression (lowess) is commonly used in cDNA microarray experiments. The background subtracted intensities were normalized by the spatial lowess method using the R add on package MAANOVA written by the Jackson Lab. For the two cDNA experiments with replicate panels within each array, we normalized the two panels separately. All control genes were excluded from data analysis for data sets AG. 


\section{Estimate of variance components}

Pair-wise comparisons among conditions/groups/treatments of gene expression levels are common goals of microarray studies. Simultaneous comparison of more than two treatments/conditions using one way ANOVA can be advantageous. However, a significant F for a comparison of several treatments does not provide information about which particular groups differ from each other. In addition, one way ANOVA is not sensitive to treatment effects when only one or two samples out of many are quite different. T-tests are commonly used to compare individual treatments in pairs.

In order to calculate power and plan sample size, one must first estimate the variance. We applied paired or two sample t-tests in this study based on the correlation between the two groups. For data set $\mathrm{A}$, as the pairs of tumor and adjacent non-tumor tissue are highly correlated, we used two tailed one sample t-tests with the normalized $\log _{2}$ ratio of tumor/non-tumor as the response variable. Data sets B-D were generated from paired samples using a reference design on cDNA arrays; Paired ttests are appropriate for these three data sets. We performed two tailed, two sample (or independent) t-tests on data sets E-G with normalized log ratio as the response variable. The two sample t-tests are based on unequal variances for the two groups of samples.

The variances of the data sets with paired samples are the variance of the difference. The common variance of the datasets with independent samples was estimated by the following formula:

$$
\text { Common variance }=\frac{s_{1}^{2}\left(n_{1}-1\right)+s_{2}^{2}\left(n_{2}-1\right)}{n_{1}+n_{2}-2}
$$

Where $\mathrm{n}_{1}, \mathrm{n}_{2}$ are the number of observations for group 1 , and group 2, respectively; and $\mathrm{S}_{1}$ and $\mathrm{S}_{2}$ are the standard deviation for group 1 , and group 2 , respectively.

To simplify power and sample size calculation, and to focus our calculation on biological variance, the log ratios of the 4 technical replicates of data sets A, E, and G were averaged for each RNA pair (data set A) or sample (data sets $E$ and $G$ ). The standard deviations across biological replicates were estimated on a gene-by-gene basis. Sample sizes were calculated using $\mathrm{R}$ for detecting a 1.5-, 2- or 4fold change for the $90 \%, 75 \%, 50 \%$, and $25 \%$ least variable genes with a range of power $(0.70,0.80,0.90)$ and confidence level $(0.01,0.001,0.0001)$, assuming equal sample size for the two groups. The numbers for sample size are rounded to integers.

\section{Authors' contributions}

JL provided the mouse liver microarray data set $\mathrm{F}$ prior to publication. CW performed all the analysis in this paper. RB supervised JL and CW and contributed to the design, coordination and writing. All authors read and approved of the final manuscript.

\section{Additional material}

\section{Additional File 1}

Sample size required to detect a 1.5-, 2-, and 4-fold changes of expression level for the $90 \%, 75 \%, 50 \%$, and $25 \%$ least variable genes for a given settings of false positive rates $(\alpha)$ and power (1- $\beta)$. This additional file shows the estimated sample size to a 1.5-, 2, and 4-fold changes of expression level for the $90 \%, 75 \%, 50 \%$, and $25 \%$ least variable genes for a given settings of false positive rates $(\alpha)$ and power (1- $\beta$ ) for all of the data sets referred in Table 1.

Click here for file

[http://www.biomedcentral.com/content/supplementary/14712164-5-87-S1.pdf]

\section{Acknowledgements}

Roger Bumgarner receives funding from the following grants: NHBLI5ROIHL072370 and IP50HL07399, NIDDK-5U24DK0588/3, NIEHSIUI 9 ESO I I 387 and NIAID-IR2 I AI052028 and 5POI AI052 I06. In addition he was partially funded by NIDA-5P30DA0 15625 during this work. Caimiao Wei is funded from grant NHBLI-5ROIHL072370. Jiangning Li was funded from NIDA grant 5P30DA015625.

\section{References}

I. Schena M, Shalon D, Davis RW, Brown PO: Quantitative monitoring of gene expression patterns with a complementary DNA microarray. Science 1995, 270:467-470.

2. Pan W, Lin J, Le CT: How many replicates of arrays are required to detect gene expression changes in microarray experiments? A mixture model approach. Genome Biol 2002, 3:research0022.

3. Lee ML, Whitmore GA: Power and sample size for DNA microarray studies. Stat Med 2002, $21: 3543-3570$.

4. Zien A, Fluck J, Zimmer R, Lengauer T: Microarrays: how many do you need? J Comput Biol 2003, I 0:653-667.

5. Pavlidis P, Li Q, Noble WS: The effect of replication on gene expression microarray experiments. Bioinformatics 2003, 19:1620-1627.

6. Smith MW, Yue ZN, Geiss GK, Sadovnikova NY, Carter VS, Boix L, Lazaro CA, Rosenberg GB, Bumgarner RE, Fausto N, Bruix J, Katze MG: Identification of novel tumor markers in hepatitis $C$ virus-associated hepatocellular carcinoma. Cancer Res 2003, 63:859-864.

7. Lapointe J, Li C, Higgins JP, van de Rijn M, Bair E, Montgomery K, Ferrari M, Egevad L, Rayford W, Bergerheim U, Ekman P, DeMarzo AM, Tibshirani R, Botstein D, Brown PO, Brooks JD, Pollack JR: Gene expression profiling identifies clinically relevant subtypes of prostate cancer. Proc Natl Acad Sci U S A 2004, 101:81 I-816.

8. Chen X, Cheung ST, So S, Fan ST, Barry C, Higgins J, Lai KM, Ji J, Dudoit S, Ng IO, Van De Rijn M, Botstein D, Brown PO: Gene expression patterns in human liver cancers. Mol Biol Cell 2002, I3:1929-1939.

9. Pritchard CC, Hsu L, Delrow J, Nelson PS: Project normal: defining normal variance in mouse gene expression. Proc Natl Acad SciU SA 2001, 98: |3266-|327|.

10. Zhao H, Langerod A, Ji Y, Nowels KW, Nesland JM, Tibshirani R, Bukholm IK, Karesen R, Botstein D, Borresen-Dale AL, Jeffrey SS: Different gene expression patterns in invasive lobular and 
ductal carcinomas of the breast. Mol Biol Cell 2004, I 5:2523-2536.

II. Dudoit S, Yang YH, Callow MJ, Speed TP: Statistical methods for identifying differentially expressed genes in replicated cDNA microarray experiments. Statistica Sinica 2002, I 2: I II-I 40.

12. Callow MJ, Dudoit S, Gong EL, Speed TP, Rubin EM: Microarray expression profiling identifies genes with altered expression in HDL-deficient mice. Genome Res 2000, 10:2022-2029.

13. Yang YH, Dudoit S, Luu P, Lin DM, Peng V, Ngai J, Speed TP: Normalization for cDNA microarray data: a robust composite method addressing single and multiple slide systematic variation. Nucleic Acids Res 2002, 30:el 5.

14. Cui $X$, Kerr MK, Churchill GA: Transformations for cDNA Microarray Data. Statistical Applications in Genetics and Molecular Biology 2003, 2:Article 4.

15. Benjamini $Y$, Hochberg $Y$ : Controlling the false discovery rate: a practical and powerful approachto multiple testing. Journal of the Royal Statistical Society Series 1995, B:289-300.

16. Kerr MK: Experimental design to make the most of microarray studies. Methods Mol Biol 2003, 224:137-I47.

17. Kerr MK, Churchill GA: Statistical design and the analysis of gene expression microarray data. Genet Res 200I, 77:123-I 28.

18. Yang $\mathrm{YH}$, Speed $\mathrm{T}$ : Design issues for cDNA microarray experiments. Nat Rev Genet 2002, 3:579-588.

Publish with Bio Med Central and every scientist can read your work free of charge

"BioMed Central will be the most significant development for disseminating the results of biomedical research in our lifetime. "

Sir Paul Nurse, Cancer Research UK

Your research papers will be:

- available free of charge to the entire biomedical community

- peer reviewed and published immediately upon acceptance

- cited in PubMed and archived on PubMed Central

- yours - you keep the copyright

Submit your manuscript here:

http://www.biomedcentral.com/info/publishing_adv.asp
BiolMedcentral 